\title{
On Exact Sequences of Quojections
}

\author{
G. Metafune and V. B. Moscatel.t.
}

\begin{abstract}
We give some general exact sequences for quojections from which many interesting representation results for standard twisted quojections can be deduced. Then the methods are also generalized to the case of nuclear Frechet spaces.
\end{abstract}

\section{INTRODUCTION}

A Fréchet space $E$ is a quojection if it is the projective limit of a sequence $\left(E_{n}, R_{n}\right)$ of Banach spaces $E_{n}$ and surjective maps $R_{n}: E_{n+1} \rightarrow E_{n}$. In this case we write $E=\operatorname{quoj}_{n}\left(E_{n}, R_{n}\right)$. A quojection is called twisted if it is not isomorphic to a countable product of Banach spaces.

It is well known that a quotient of a quojection is again a quojection (possibly Banach). Here we want to investigate quotients of products with respect to quojection subspaces with regard to the property of being twisted or not. This has an intrinsic interest as well as implications to the existence or not of unconditional bases. It is clear that the problem is equivalent to the study of short exact sequences of quojections of the form

$$
0 \rightarrow F \rightarrow G \rightarrow E \rightarrow 0
$$

where, in our case, $G$ is a product. Thus, in $\$ 1$ we characterize the vanishing of the first derived functor $\operatorname{Ext}^{\prime}(E, F)$, which is equivalent, for fixed $E$ and $F$. to the splitting of any exact sequence as $\left(^{*}\right)$. To introduce the argument we show that, for any given quojection $E$, there always exist a quojection $F$ and a cardinal $d$ for which we have an exact sequence as (*) with $G=\left(l_{d}^{l}\right)^{\mathrm{N}}$. This 
could also be deduced by carrying on further the proof of the result in [1], but, for the convenience of the reader (and because the effort is the same), we prefer to give here the full proof. In $\$ 2$ we then give a general exact sequence for standard quojections. Based on the fact, proved in [16], that if $E$ or $F$ are standard twisted quojections, then no sequence (*) can split, in $\$ 3$ we are able to obtain from our general sequence many interesting representation results involving standard twisted quojection and, consequently, unconditional bases. Last, but not least, applications are made in $\$ 4$ to the case of nuclear spaces.

The notation we follow is standard, but, for brevity's sake, we use the term "surjection" to mean a linear and continuous surjective map.

\section{ON THE VANISHING OF THE FUNCTOR Ext ${ }^{\prime}$ FOR QUOJECTIONS}

Theorem 1.1. If $E$ is a quojection, then there is a cardinal d and $a$ surjection $T:\left(l_{1}^{1}\right)^{N}-$ E such that ker $T$ is a quojection.

Proof. By definition we have $E=$ quoj $_{n}\left(E_{n}, R_{n}\right)$, where the $E_{n}$ are Banach spaces and the maps $R_{n}: E_{n+1} \rightarrow E_{n}$ are surjective. Let $d \geq \operatorname{dens}(E)$ and let $T_{1}: I_{d}^{l} \rightarrow E_{1}$ be a surjection. Supposing that $T_{n}:\left(I_{d}^{l}\right)^{n} \rightarrow E_{n}$ has been defined, let $\tilde{T}_{n}$ be a lifting of $T_{n}$ into $E_{n+1}$ (i.e., $R_{n} \cdot \tilde{T}_{n}=T_{n}$ ), let $S_{n}$ : $I_{d}^{1} \rightarrow$ ker $R_{n}$ be a surjection and define $T_{n+1}:\left(l_{d}^{l}\right)^{n} \times l_{d}^{l} \rightarrow E_{n+1}$ by $T_{n+1}=\tilde{T}_{n}+S_{n t}$. The required map is then obtained by setting $T\left(x_{n}\right)=\left(T_{n}\left(x_{1}, \ldots, x_{n}\right)\right)_{n}$ for $\left(x_{n}\right) \in$ $\left(l_{d}^{l}\right)^{\mathrm{N}}$. For the surjectivity, take any $\left(y_{n}\right) \in E_{\text {; }}$ since $y_{1} \in E_{1}$ there is $x_{1} \in l_{d}^{\prime}$ with $y_{1}=T_{1} x_{1}=R_{1} \tilde{T}_{1} x_{1}$. But $y_{1}=R_{1} y_{2}$ with $y_{2} \in E_{2}$, hence $y_{2}-\tilde{T}_{1} x_{1}=S_{1} x_{2}$ for some $x_{2} \in I_{d}^{1}$, i.e. $l_{2}=T_{2}\left(x_{1}, x_{2}\right)$. Inductively we obtain $v_{n}=T_{n}\left(x_{1}, \ldots, x_{n}\right)$ for all $n$, hence $\left(y_{n}\right)=T\left(x_{n}\right)$ with $\left(x_{n}\right) \in\left(l_{d}^{l}\right)^{\mathrm{N}}$. It remains to show that ker $T$ is a quojection.

For each $n$ let $P_{n}:\left(l_{d}^{l}\right)^{n+1} \rightarrow\left(l_{d}^{l}\right)^{n}$ be the canonical projection; then ker $T=\operatorname{proj}_{n}\left(\operatorname{ker} T_{n}, P_{n}\right)$. Let $z=\left(x_{1}, \ldots, x_{n}\right) \in \operatorname{ker} T_{n}$; then $0=T_{n} z=R_{n} \tilde{T}_{n} z$ implies $\tilde{T}_{n} z \in \operatorname{ker} R_{n}$ and we may choose $x_{n+1} \in l_{d}^{\prime}$ with $\tilde{T}_{n} z+S_{n} x_{n+1}=0$. This means $\left(z, x_{n+1}\right) \in \operatorname{ker} T_{n+1}$ hence each $P_{n}: \operatorname{ker} T_{n+1} \rightarrow \operatorname{ker} T_{n}$ is surjective and ker $T$ is a quojection, as claimed.

Theorem I.I is the analogue for quojections of the classical result for Banach spaces. However, the analogy stops here. In fact, while for a separable Banach space $X$ not isomorphic to $/$ there is a unique, up to an 
automorphism of $l$, surjection $T: I^{\prime} \rightarrow X(\mathrm{cl}$. [10, I, Theorem 2.f.8., p. 108]). so that the kernels of all such maps $T$ are isomorphic, for quojections the situation is completely different, as the following example shows.

Example 1.2. I..et $T:(/ /)^{N} \rightarrow(R)^{N}$ be the canonical surjection ind uced by the quotient map $q: /^{1} \rightarrow /^{2}$. Clearly, ker $T=(\operatorname{ker} q)^{N}$ and so $\operatorname{ker} T$ is a quojection. Now let $N$ be a nuclear Fréchet space not isomorphic to $\omega$; by [II, Proposition 1. 14] $\left(/^{2}\right)^{\mathrm{N}} / N$ is isomorphic to $\left(/^{2}\right)^{N}$ and we denote by $J$ one such isomorphism. It follows that, if $Q:\left(/^{2}\right)^{N} \rightarrow\left(/^{2}\right)^{N} / N$ is the quotient map, then the map $S=J Q T$ is a continuous surjection of $(/ /)^{N}$ onto $\left(/^{2}\right)^{N}$ for which $\operatorname{ker} S\left(=T^{-1}(N)\right.$ is not a quojection, hence not isomorphic to $\operatorname{ker} T$.

Remark 1.3. At this stage one might think that the kernels of two maps from $(/ /)^{N}$ onto the same separable quojection (not isomorphic to a complemented subspace of a product of the form $X \times(/ /)^{\text {N }}$ with $X$ Banach) would have to be isomorphic if they were both quojections. However, not even this is true, as will be shown in Example 3.13 below.

In the rest of this section we shall discuss some consequences of Theorem 1.1 and related results. We recall that the vanishing of the first derived functor $\operatorname{Ext}^{\prime}(E, F)$ for Fréchet spaces $E$ and $F$ means that every exact sequence

$$
0 \rightarrow F \rightarrow G \rightarrow E \rightarrow 0
$$

splits and, consequently, $G \simeq E \oplus F$. For a discussion of this topic see [20].

Proposition 1.4. Let E be a quojection. The following are equivalent:

(i) $\operatorname{Ext}^{1}(E, F)=0$ for every quojection $F$;

(ii) $E$ is isomorphic to a complemented subspace of $\left(l_{d}^{l}\right)^{\mathrm{N}}$ for some cardinal $d$ (and hence either $\mathrm{E} \simeq l_{d^{\prime}}^{\prime}, \omega, l_{d^{\prime}}^{\prime} \times \omega\left(\mathrm{d}^{\prime} \leq \mathrm{d}\right)$ or $E$ contains a complemented copl of $\left.\left(/^{\prime}\right)^{\mathrm{i}}\right)$.

Proof. (i) $\rightarrow$ (ii): By Theorem 1.1 there is an exact sequence

$$
0 \rightarrow k e r T \rightarrow\left(l_{d}\right)^{\mathrm{N}} \stackrel{T}{\rightarrow} E \rightarrow 0
$$

and by assumption this sequence splits. Then the assertion in brackets follows essentially from the proof of Theorem 1.2 in [15]

(ii) $\rightarrow$ (i): This is a consequence of [20, Remark b), pag. 173]. 
Remark 1.5. In the separable case, assertion (ii) above may be replaced by $E \simeq I^{\prime}, \omega, l^{\prime} \times \omega$ or $(/)^{N}(\mathrm{c} f .[15$, Theorem 1.2$])$.

Proposition 1.6. Let F be a Frechet sperce. The following are equivalent:

(i) $\operatorname{Ext}^{\prime}(E, F)=0$ for ewery quojection $E$ :

(ii) $F$ is isomorphic to a complemented subspace of $\left(l_{d}^{\infty}\right)^{\mathrm{N}}$ for some cardinal $d$ (and hence cither $F \simeq X, \omega, X \times \omega(X$ an injertive Banach spare $)$ or $F$ contains a complemented copy of $\left.\left(l^{x}\right)^{N}\right)$.

Proof. (i) $\rightarrow$ (ii): Let $d$ be such that $F$ is a subspace of $\left(i_{d}^{x}\right)^{x}$. By assumption the exact sequence

$$
0-\Gamma \rightarrow\left(l_{d i}^{\infty}\right)^{\mathrm{N}} \rightarrow\left(l_{d}^{\infty}\right)^{\mathrm{N}} / F \rightarrow 0
$$

splits and (ii) follows from [1. 15. Proposition 3.8 or Propisition 3.14].

(ii) $\rightarrow$ (i): This is immediate, since $F$ is injective.

Proposition 1.7. Let F be a Frechel space. The following are equivalent:

(i) $E_{x}\left(\left(I_{d}^{1}\right)^{x}, F\right)=0$ for everv cardinal d:

(ii) $E \times H^{\prime}\left(\left(O^{\mathrm{l}}\right)^{\mathrm{r}}, F\right)=0$;

(iii) $\operatorname{Ex}^{\prime}(\omega, F)=0$;

(iv) Fis a quojection.

Proof. (i) $\rightarrow$ (ii) $\rightarrow$ (iii): Clear.

(iii) $\rightarrow$ (iv): By [19, Theorem 5.2].

(iv) - (i): By Proposition 1.4.

Proposition 1.8. Let F be a separable Fréchet spare. The following are equivalen:

(i) $\mathrm{Ext}^{1}\left(l^{1}, F\right)=0$;

(ii) Fis quasinornable. 
Proof. First of all, observe that $\operatorname{Ext}^{\prime}\left(l^{\prime}, F\right)=0$ is quickly seen to be equivalent to the following: for any exact sequence

$$
0 \rightarrow F \rightarrow G \stackrel{q}{\rightarrow} E \rightarrow 0
$$

with $G$ separable and Fréchet and $F=$ ker $q$. every map $T: l^{\prime}-E$ can be lifted to a map $\widetilde{T}: l^{1} \rightarrow G$ such that $q \tilde{T}=7$. In turn, this happens if and only if $q$ lifts the bounded subsets of $E$ to bounded subsets of $G$. Thus it suffices to show that $F$ is quasinormable if and only if for any sequence (1) the map $q$ lifts the bounded subsets. Now if $F$ is quasinormable, then $q$ lifts the bounded subsets by [4, Proposition 2]. Conversely, if this is the case, represent $F$ as a reduced projective limit $F=\operatorname{proj}_{n} F$ of Banach spaces $F_{n}$. By [20, Lemma I.l] we have an exact sequence

$$
\left.\left.0 \rightarrow F \rightarrow\right|_{n} F_{n} \stackrel{a}{-}\right|_{n} F_{n} \rightarrow 0
$$

Since $q$ lifts the bounded subsets by assumption and $\prod_{n} F_{n}$ is quasinormable by [3, Theorem, Pag. 159] (cfr. also [5], Pag. 33) $F$ is quasinormable. Note that in general $F$ is quasinormable if and only if Ext $\left(l_{t}^{\prime}, F\right)=0$ with $d=\operatorname{dens}(F)$.

We conclude this section by showing that Proposition 1.4 cannot be improved by relaxing the condition that $F$ be a quojection. In other words, from $(l)^{\mathrm{N}}$ being a quotient it does not follow, in gencral, that it is also a complemented subspace. At the same time, this will show that Proposition 3.3 and Corollary 3.4 of [15] do not hold for quojections. Precisely, we now exhibit examples of separable quojections having $(/)^{N}$ as a quotient but not even as a subspace.

Example 1.9. Let $X$ be a separable Banach space such that no power $X^{n}$ contains an isomorphic copy of $/ l$ (e.g., $X=c_{n}$ or $l^{\prime \prime}, 1<p<\infty$ ) and put $E=l^{\prime} \times X^{\mathrm{N}}$. Next, choose a biorthogonal system $\left\{\left(y_{n}, y_{n}^{\prime}\right): y_{n} \in X, y_{n}^{\prime} \in X^{\prime}\right\}$ with $\left(y_{n}^{\prime}\right)$ total over $X$ and $\left\|y_{n}^{\prime}\right\|=1$.

Then, define a map $i: X \rightarrow h^{\prime}$ by $i(x)=\left(2^{-\mu}<x, y_{n}^{\prime}>\right)$; clearly $i$ is continuous and has a dense range. Now write $l^{\prime}$ in $E$ as $I^{\prime}(/)$ and define $T: E \rightarrow\left(l^{\prime}\right)^{N}$ by $T\left(\left(a_{n}\right),\left(x_{n}\right)\right)=\left(a_{n}+i\left(x_{n}\right)\right)$ for $\left(a_{n}\right) \in l^{\prime}\left(l^{\prime}\right)$ and $\left(x_{n}\right) \in X^{N}$. T is continuous and surjective: in fact, given $\left(b_{n}\right) \in\left(l^{\prime}\right)^{\mathrm{N}}$, choose $\left(x_{n}\right) \in X^{\mathrm{N}}$ so that $\left(b_{n}-i\left(x_{n}\right)\right) \in l^{\prime}\left(l^{\prime}\right)$; if $a_{n}=b_{n}-i\left(x_{n}\right)$, then $T\left(\left(a_{n}\right),\left(x_{n}\right)\right)=\left(b_{n}\right)$. Thus $E$ has at quotient isomorphic to $(l)^{\mathrm{N}}$. We show that no subspace of $E$ is isomorphic to $\left(l^{\prime}\right)^{\mathrm{N}}$.

Arguing by contradiction. suppose that there exists an isomorphism $/$ of $\left(I^{\prime}\right)^{N}$ into $E$. For $\left(a,\left(x_{n}\right)\right) \in E$, with $a \in I^{\prime}$ and $\left(x_{n}\right) \in X^{N}$, put

$$
p_{1}\left(a,\left(x_{1}\right)\right)=\|a\|_{1}
$$


and

$$
p_{k+1}\left(a,\left(x_{n}\right)\right)=\|a\|_{1}+\sum_{n=1}^{h}\left\|x_{n}\right\|_{x} .
$$

Also. For $\left(a_{\prime \prime}\right) \in(/ 1)^{\mathrm{N}}$ put

$$
q_{k}\left(a_{n}\right)=\sum_{n}^{k}\left\|a_{n}\right\|_{1}
$$

Then $\left(p_{k}\right)$ and $\left(q_{k}\right)$ are lundamental sequences of seminorms for $E$ and $\left(l^{\prime}\right)^{\mathrm{N}}$ respectively and for any $b \in\left(l^{\prime}\right)^{N}$ we must have

$$
c_{1} p_{1}(J / h) \leq q_{k}(h) \leq q_{h+1}(h) \leq c_{2} \eta_{i+1}(J h) \leq c_{3} q_{m}(h)
$$

for suilable positive constants $c_{1}, c_{2}, c_{3}$ and integers $k \leq j<m$. If $d_{1}$ and $d_{2}$ are the restrictions of $J$ to $(/)^{k}$ and $(/)^{\prime \prime \prime}$ respectively, from (2) we obtain the following diagram

$$
\begin{aligned}
& \left(l^{1}\right)^{m} \stackrel{\stackrel{\prime}{\rightarrow}}{\rightarrow} /^{1} \times X^{i} \\
& Q \downarrow+l{ }_{(l)}^{k} \underset{\vec{J}_{1}}{\rightarrow} I^{\prime}
\end{aligned}
$$

where $P$, resp. $Q$, is the projection onto the first component, resp. the lirst $k$ components, and $P J_{2} b=J_{1} Q b$ for all $b \in\left(l_{1}\right)^{m}$. Now consider the $(k+1)-$ st copy of $l^{\prime}$ in $(l)^{m}$ and denote it by $l_{k+1}^{\prime}$. It Collows from (2) that $J_{2}$ is an isomorphism on $l_{k+1}^{1}$. But if $b \in l_{k+1}^{1}$, then $Q b=0$, hence $P J_{2} b=0$ and, therefore, $J_{2} b \in X^{i}$. However, this contradicts our assumption on $X$.

Note that, with reference to the above example, it follows from Proposition 1.4 that for any surjection $S: E-\left(l^{1}\right)^{N}, \operatorname{ker} S$ is not a quojection. It is also follows from theorem 1.1 that every separable quojection is a quotient of $E$.

Finally, we observe that Theorem 1.1 has as a consequence also the following:

Proposition 1.10. Every strict (LB)-space $G$ is a subspace of $\left(l_{d}^{\infty}\right)^{N}$ for some cardinal d.

Proof. $G^{\prime}$ is a quojection and hence a quotient of $\left(l_{d}^{\prime}\right)^{N}$ with respect to a quojection subspace, by Theorem 1.1. Since the quotient map lifts the bounded subsets, $G^{\prime \prime}$ is a subspace of $\left(I_{d}^{p}\right)^{\mathrm{N}}$, whence so is $G$. 


\section{AN EXACT SEQUENCE FOR STANDARD QUOJECTIONS}

'Jheorem I. 1 of the previous section tells us that every yuojection lican be realized as the quotient of $\left(l_{d}^{l}\right)^{\mathrm{N}}$ by a quojection subspace $f$. Now, in general. even if $E$ is given. there is nothing we can say about $F$ or about the possibility of replacing $(/ d)^{N}$ by some other product (depending on $l:$ ol course). This is due to our lack of knowledge about general yuojections, There is, however. an important class of quojections for which we can say a greal deal and which we shall texamine in this section.

We recill that at present there are only two methods for constructing twisted quojections, plus an exceptional case discussed in [16,\$2]. The lïst was originally devised in [17]: because of its semplicity and versatility. it is still the major source of examples and counterexamples. The second, treated in [12], is much less concrete and, so far, it has been ol very litte use. Therefore, it is not surprising that we shall appeal once again to the former method. In doing so, we find it convenient to introduce a specilic notation, for which we recall the construction in [17] in the lollowing form.

Let $\left(X_{n}\right)$ and $\left(Z_{n}\right)$ be two sequences of Banach spaces for which there are surjections $s_{n}: X_{n} \rightarrow Z_{n}$. II $L$ is a Banach sequence space, we form the steps

$$
F_{1}=\left(\underset{n}{\oplus} Z_{n}\right)_{L}=L\left(Z_{n}\right)
$$

and, for all $k$.

$$
\left.F_{k+1}=\left[\left(\oplus_{n \leq k} X_{n}\right) \oplus \underset{n>k}{\oplus} Z_{n}\right)\right]=L\left[\left(X_{n}\right)_{n \leq k^{\prime}}\left(Z_{n}\right)_{n>k}\right] .
$$

Clearly the maps $s_{n}$ induce, together with the identies of $X_{n}$ and $Z_{n}$. surjections $S_{h}: F_{k+1} \rightarrow F_{k}$. The projective limit of the sequence $\left(F_{h}, S_{h}\right)$ is. therefore, a quojection, which we shall denote by $Q\left[\left(X_{n}\right),\left(Z_{n}\right),\left(s_{n}\right) ; L\right]$. Such quojections form a subclass of the class of Fréchet spaces defined in [2] and we shall refer to quojections isomorphic to them as standard quojections. Note that $Q\left[\left(X_{n}\right),(\{0\}),(0) ; L\right]=|| X_{n}$ so that every countable product of Banach spaces is a standard quojection.

The above construction hals the added advantage that it may be performed also when the $X_{n}, Z_{n}$ and $L$ are Fréchet spaces (as implicitly done in [17]) and we shall give an interesting application of this in the last section.

If the spaces $Z_{n}$ above are quotients of the $X_{n}$ with respect to closed subspaces $Y_{n}$ and if the $s_{n}$ are the quotient maps, the corresponding standard quojection will de denoted by $Q\left[\left(X_{n}\right),\left(X_{n} / Y_{n}\right) ; L\right]$ if no conlusion is likely to arise. Clearly. we always have

$$
Q\left[\left(X_{n}\right),\left(Z_{n}\right),\left(s_{n}\right) ; L\right] \simeq Q\left[\left(X_{n}\right),\left(X_{n} / \text { ker } s_{n}\right) ; L\right],
$$


To end our preparations, we find it useful to state the following lemma, which is essentially Theorem 1.1 of [16] (the "only if part of the lemma being obvious).

Lemma 2.1. $Q\left[\left(X_{n}\right),\left(Z_{n}\right),\left(S_{n}\right): L\right]$ is awisted if and only if ker $s_{n}$ is not complemened in $X_{n}$ for 'infinitely many' $n$

Now the proof of our main result of this section begins with the following lemmit, which is at the heart of the matter.

Lemma 2.2. Let $Q\left[\left(X_{n}\right),\left(X_{n} / Y_{n}\right) ; L\right]$ be any standard quojection and let $\left(Z_{n}\right)$ he a sequence of Banach spaces admituing a sequence $\left(f_{n}\right)$ of contimus linear maps. $f_{n}: Z_{n} \rightarrow Y_{n}$ with dense ranges. Then there is a surjection $S: L\left(X_{n}\right) \times|| Z_{n} \rightarrow Q\left[\left(X_{n}\right),\left(X_{n} / Y_{n}\right), L\right]$ such that ker $S$ is a Fréchet space of Moscatelli trpe (cfr. [2, Definition 1.3]).

Proof. First of all, put $E=Q\left[\left(X_{n}\right),\left(X_{n} / Y_{m}\right): L\right]$ and observe that, by $[2$. Proposition 1.4], algebraically we have

$$
E=\left\{\left.\left(x_{n}\right) \in\right|_{n} \mid X_{n}:\left(q_{n}\left(x_{n}\right)\right) \in L\left(X_{n} / Y_{n}\right)\right\} .
$$

where $q_{n}: X_{n} \rightarrow X_{n} / Y_{n}$ is the quotient map for every $n$. Moreover. the topology of $E$ may be defined by the system $\left(f_{k}\right)$ of seminorms given by

$$
\begin{aligned}
& p_{1}\left(x_{n}\right)=\left\|\left(\left\|q_{n}\left(x_{n}\right)\right\|_{x_{n} / r_{n}}\right)\right\|_{L}, \\
& p_{k+1}\left(x_{n}\right)=p_{1}\left(x_{n}\right)+\sum_{n=1}^{k}\left\|x_{n}\right\|_{x_{n}} .
\end{aligned}
$$

Now for $\left(\left(x_{n}\right),\left(z_{n}\right)\right) \in L\left(X_{n}\right) \times\left.\right|_{n} Z_{n}$ the equation

$$
S\left(\left(x_{n}\right),\left(z_{n}\right)\right)=\left(x_{n}+f_{n}\left(z_{n}\right)\right)
$$

defines a linear map $S: L\left(X_{n}\right) \times\left\|_{n} Z_{n} \rightarrow\right\|_{n} X_{n}$. Using (3) and (4) it is easy to see that $S$ lakes its values in $E$ and is continuous as a map into $E$; hence, it remains to prove that $S$ is surjective and ker $S$ is of the required type.

Let $\left(x_{n}\right) \in E$, by $(3)$ there is $\left(v_{n}\right) \in L\left(X_{n}\right)$ with $q\left(v_{n}\right)=q_{n}\left(x_{n}\right)$ for all $n$. Put $l_{n}=x_{n}-v_{n}$ : since $l_{n} \in Y_{n}$ and $f_{n}$ has a dense range, we can find $z_{n} \in Z_{n}$ such that $\left(l_{n}-f_{n}\left(z_{n}\right)\right) \in l\left(Y_{n}\right)$. Thus, if $u_{n}=v_{n}+l_{n}-f\left(z_{n}\right)$ for all $n$. then $\left(u_{n}\right) \in I .\left(X_{n}\right)$ and $S\left(\left(u_{11}\right),\left(z_{1,}\right)\right)=\left(x_{11}\right)$. 
Finally, by (5) we have

$$
\operatorname{ker} S=\left\{\left(\left(-f_{n}\left(z_{n}\right)\right),\left(z_{n}\right)\right):\left(z_{n}\right) \in|| Z_{n},\left(f_{n}\left(z_{n}\right)\right) \in L\left(Y_{n}\right)\right\}
$$

from which the assertion about ker $S$ follows, again by [2, Proposition 1.4].

Theorem 2.3. Under the assumptions of Lemma 2.2 suppose that the maps. $f_{n}$ can be chosen wo be surjections and denote them by $s_{n}$. Then

$$
\text { ker } S \simeq Q\left[\left(Z_{n}\right),\left(Y_{n}\right),\left(s_{n}\right) ; L\right] \text {. }
$$

Consequently:

(a) ker $S$ is Banach if and only if ker $s_{n}=0$ for all but finitely many n;

(b) ker $S \simeq \omega \times$ Banach if and only if $0<\operatorname{dim}\left(\right.$ ker $\left.s_{n}\right)<\infty$ for all but finitely many $n$;

(c) ker $S$ is twisted if and only if ker $s_{n}$ is not conplemented in $Z_{n}$ for infinitely mant: $n$.

Moreover, we have established the existence of an exact sequence

$$
O \rightarrow Q\left[\left(Z_{n}\right),\left(Y_{n}\right),\left(s_{n}\right): L\right] \rightarrow L\left(X_{n}\right) \times\left.\right|_{n} Z_{n} \rightarrow Q\left[\left(X_{n}\right),\left(X_{n} / Y_{n}\right): L\right] \rightarrow 0 .
$$

Proof. (7) follows from (6) and Proposition 1,4 of [2], while (8) follows from (7) and Lemma 2.2. It is then an easy matter to verify that (a), (b) and (c) hold (for (c) use Lemma 2.1).

The above theorem has many interesting corollaries, which will be given in the next section. Here we conclude with the following consequence of L.emma 2.2 .

Propesition 2.4. Let E be any standard quojection of the form $Q\left[\left(X_{n}\right)\right.$. $\left.\left(X_{n} / Y_{n}\right) ; H^{\prime}\right]$, with $Y_{n}$ separable for all $n$. Then for every sequence $\left(Z_{n}\right)$ of Banach spaces there is a surjection $S: I^{\prime}\left(X_{n}\right) \times\left.\right|_{n} Z_{n} \rightarrow E$ such that ker $S$ is not distinguished (hence not quasinormable). In particular, this holds for $E=\left.\right|_{n} Y_{n}$

Proof. For each $n$ take a compact map $f_{n}: Z_{n} \rightarrow Y_{n}$ with a dense range $\left(f_{n}\right.$ may be constructed more or less as $i$ was in Example 1.9). Then the surjection 
$S$ constructed in the proof of Lemma 2.2 meets the requirement, by $[2$, Corollary 2.5]

Remark 2.5. It is interesting to note that, if in the above proposition we take $X_{n}=Y_{n}=Z_{n}=l^{\prime}$ and $f_{n}=f$ for all $n$, with $f^{\prime}\left(a_{k}\right)=\left(k^{-1} a_{k}\right)$ for $\left(a_{k}\right) \in l^{1}$, then for the corresponding surjection $S:(/ /)^{N} \rightarrow(/)^{N}$ we find that ker $S$ is the non-distinguished Fréchet space of Köthe-Grothendieck (cfr. [9, \$31, 7, pag. $435]$ ).

\section{CONSEQUENCES}

This section is devoted to the investigation of various consequences of Theorem 2.3. Here "product" means a countable (and not finite) product of Banach spaces. Moreover, whenever all the Banach spaces $X_{n}$ (resp., $Z_{n}$ ) and maps $s_{n}$ are taken equal to one fixed space $X$ (resp., $Z$ ) and map $s$, we simply write $Q(X, Z, s ; L)$ for the corresponding standard quojection.

The first consequence of Theorem 2.3 is the following quite strange

Corollary 3.1. Every standard twisted quojection is isomorphic to the quotient of a product by a Banach space.

Proof. Taking in Theorem 2.3 $Z_{n}=Y_{n}$ and $s_{n}=$ the identity map of $Y_{n}$ for all $n$, we obtain from (8) an exact sequence

$$
0 \rightarrow L\left(Y_{n}\right)-L\left(X_{n}\right) \times\left.\right|_{n} Y_{n} \rightarrow Q\left[\left(X_{n}\right),\left(X_{n} / Y_{n}\right) ; L\right] \rightarrow 0 .
$$

It is clear that we can also represent a standard twisted quojection as the quotient of a product by a product. Consequently, since a standard twisted quojection is never complemented in a product [16. Theorem 1.5 (b)], we have

Corollary 3.2. Let E be any standard wisted quojection. Then there is Banach space $X$ and a product $F$ such that

$$
\operatorname{Ext}^{\prime}(E, X) \neq 0 \text { and } \operatorname{Ext}^{\prime}(E, F) \neq 0 \text {. }
$$

Next, we observe that Corollary 3.1 has the following counterpart.

Corollary 3.3. Every standard twisted quojection can be embedded as subspace $F$ of a product $G$ such that $G / F$ is again a product. 
Proof. Represent the given quojection as $Q\left[\left(Z_{n}\right),\left(Y_{n}\right),\left(s_{n}\right) ; L\right]$. put $X_{n}=Y_{n}$ for all $n$ and apply (8) to obtain an exact sequence

$$
0 \rightarrow Q\left[\left(Z_{n}\right),\left(Y_{n}\right),\left(s_{n}\right) ; L\right] \rightarrow L\left(Y_{n}\right) \times\left.\right|_{n} Z_{n} \rightarrow \|_{n} Y_{n} \rightarrow 0 .
$$

Corollary 3.4. Let $F$ be any standard twisted quojection. Then there exists a product Esuch that $\operatorname{Ext}^{1}(E, F) \neq 0$.

Here we pause to note that it is not possible to obtain an exact counterpart of Corollary 3.I, namely, the conclusion of Corollary 3.3 cannot be improved to " $G / F$ is Banach" on account of the following

Proposition 3.5. Let E be a quojection and let F be a subspace of Estuch that $E / F$ is Banach. Then $F$ is a quojection. Moreover. if $E$ is a product, then also $F$ is a product.

Proof. Let $E=\operatorname{quoj}_{n}\left(E_{n}, R_{n}\right)$ and write $F$ as the rectuced projective limit $F=\operatorname{proj}_{n}\left(F_{n}, R_{n}\right)$, where each $F_{n}$ is a closed subspace of $E_{n}$. Then $E / F=$ quoj, $_{n}$ $\left(E_{n} / F_{n}, \bar{R}_{n}\right)$, where the maps $\bar{R}_{n}: E_{n+1} / F_{n+1} \rightarrow E_{n} / F_{n}$ are induced by the maps $R_{n}: E_{n+1} \rightarrow E_{n}$. If $E / F$ is Banach, we may assume that each $\bar{R}_{n}$ is an isomorphism. In particular, for all $n$.

$$
0=\operatorname{ker} \bar{R}_{n}=\left(R_{n}^{-1}\left(F_{n}\right)+F_{n+1}\right) / F_{n+1} \cdot
$$

which implies that in $E_{n+1}$ we must have $R_{n}^{-1}\left(F_{n}\right) \subset F_{n+1}$. Hence $F=\operatorname{quoj}_{n}\left(F_{n}, R_{n}\right)$.

Suppose now that $E$ is a product ||$X_{k}$ of Banach spaces $X_{k}$ and, for each $n$, let $R_{n}:\left.\sum_{k=1}^{n+1} X_{k} \rightarrow\right|_{k=1} ^{n} X_{k}$ be the canonical projection. With $F_{1} \subset X_{1}$, from $R_{1}^{-1}$ $\left(F_{1}\right)=F_{2}$ we obtain $F_{2}=F_{1} \times X_{2}$ and, in general, $F_{n}=F_{1} \times\left.\right|_{k=2} ^{n} X_{k}(n>1)$, so that $F$ is a product, as claimed.

Remark 3.6. Note that $F$ may be a product even if $E$ is twisted, as shown in [11, Corollary 2.3].

Going back to the consequences of Theorem 2.3, now we have

Corollary 3.7. Every quojection $Q\left[\left(X_{n}\right),\left(X_{n} / Y_{n}\right) ; L\right]$, with $Y_{n} \neq l_{d l}^{\prime}$ for infinitely many $n$ and all cardinal numbers $d$, is isomorphic to the quotient of 
a product by a standard twisted quojection. In particular, the conchusion holds for every product of Banach spaces $Y_{n}$ as above (cfr. (10)).

Proof. Take $d \geq \operatorname{dens}\left(Y_{n}\right)$ for all $n$, then choose surjections $s_{n}: I_{i}^{l} \rightarrow Y_{n}$ and apply Theorem $2.3(\mathrm{c})$ with $Z_{n}=l_{d}^{\prime}$.

Corollary 3.8. Let $\left(Y_{n}\right)$ be a sequence of Banadh spaces and suppose that there are two infinite subsets $N_{1} . N_{2}$ of $\mathbf{N}$ such that, if $n \in N_{1}, Y_{n}$ is not injective and, if $n \in N_{2}, Y_{n}$ is not projective. Put

$$
E=Q\left[\left(X_{n}\right),\left(X_{n} / Y_{n}\right) ; L\right] \text { and } F=Q\left[\left(Z_{n}\right),\left(Y_{n}\right),\left(s_{n}\right) ; L\right] \text {. }
$$

Then, given any wisted E (resp., F) there exist a twisted F (resp., E) and a preduct G for which we have an exact sequence

$$
0 \rightarrow F \rightarrow G \rightarrow E \rightarrow 0
$$

and, consequendly, $\operatorname{Ext}^{1}(E, F) \neq 0$.

We note the following special case of Corollary 3.8 (or 3.7). which should be compared with Theorem 1.1 .

Corollary 3.9. Let $Y$ be a sutspace of $/ 1$ with $Y \neq l^{\prime}$ and let $x: I^{\prime}-Y$ be a surjection. Then there is an exact sequence

$$
0 \rightarrow Q\left(I^{\prime}, Y, s ; I^{\prime}\right) \rightarrow\left(I^{\prime}\right)^{N} \rightarrow Q\left(I^{\prime}, I / Y ; I^{\prime}\right) \rightarrow 0 .
$$

where, of course, both quojections are twisted.

Remark 3.10. We do not know if the above result holds with ${ }^{\prime}$ replaced by $l^{\prime}(1<p<\infty, p \neq 2)$. It is easy to see that it holds for $p^{\infty}$ and $c_{c}$ and also for $C^{\prime}([0.1])$ and $L^{\prime}(0,1)$, with appropriate choices of $Y$, but again we know nothing about the case of $L^{\prime \prime}(0,1)(1<p<\infty, p \neq 2)$. However, it calmost" holds for $L^{\prime}(0,1)$ in the sense that we have, putting $L^{\prime}(0,1)=L^{t}$ for all $1<1<\infty$,

Corollary 3.11. Let $p, q, r, s$ be positive real numbers satisfing

$$
1<p<q<2<r<s<\infty
$$


and let $L$ be reflexive. Then the reflexive product

$$
G=L\left(L^{\prime \prime} \oplus L^{\prime}\right) \times\left(L^{\prime \prime} \oplus L^{\prime}\right)^{N}
$$

contains a standard twisted quojection $F$ such that also $G / F$ is a standard misted quojection.

Proof. By [10, II. Corollary 2.f.5, p. 212] $L^{q}$ is (isometrically) a subspace of $L^{\prime \prime}$, whence $L^{\prime}$ is a quotient of $L^{\prime}$. Since $l^{\prime}$ is a subspace of $L^{\prime}$, we see from [8. Corollary 3, p. 168] that $L^{\prime}$ cannot be isomorphic to a subspace of $L$. This implics also that $L^{q}$ cannot be isomorphic to a complemented subspace of $L^{\prime}$. Now observe that $L^{\prime \prime} \oplus L^{\prime} / L^{\prime \prime} \oplus L^{r}=L^{\prime \prime} / L^{\prime \prime}$ and form the standard quojection $E=Q\left(L^{\prime \prime} \oplus L^{\prime}, L^{\prime \prime} / L^{4} ; L\right)$. Then, by Theorem 2.3 (or Corollary 3.8), $E \simeq G / F$, where $F \simeq Q\left(L^{4} \oplus L^{\prime}, L^{4} \oplus L^{r}, s ; L\right)$ (here $s: L^{4} \oplus L^{\prime} \rightarrow L^{4} \oplus L^{r}$ is the map which is the identity on $L^{4}$ and the quotient map on $L^{\prime}$ ).

Clearly the above result holds also with $L^{q}$ and $L^{r}$ replated by $I^{t}$ and $I^{\prime}$ respectively. Moreover, an application of (9) and (10) establishes the following particular case of Corollaries 3.1 and 3.3 which we find worth mentioning because all the spaces involved are reflexive.

Corollary 3.12. There are exact sequences

$$
\begin{aligned}
& 0 \rightarrow L^{\prime \prime} \rightarrow I^{\prime \prime}\left(L^{\prime \prime}\right) \times\left(L^{\prime \prime}\right)^{\mathrm{N}}-Q\left(L^{\prime \prime}, L^{\prime \prime} / L^{\prime} ; H^{4}\right) \rightarrow 0, \\
& 0 \rightarrow Q\left(L^{s}, L^{r} ; l^{\prime}\right) \rightarrow I^{\prime} \times(I .)^{\mathrm{N}} \rightarrow\left(I^{\prime}\right)^{\mathrm{N}} \rightarrow 0 .
\end{aligned}
$$

where $1<p<q<2<r<s<\infty$.

We terminate our list of direct consequences of Theorem 2.3 by giving the example promised in Remark 1.3.

Example 3.13. Let $X=C([0,1])$ and $E=Q\left(X, X / I^{\prime} ; /\right)$. From Theorem 2.3 (a) we obtain a surjection $S_{1}: I^{\prime}(X) \times\left(l^{\prime}\right)^{\mathrm{N}} \rightarrow E$ with ker $S_{1} \simeq l^{\prime}$. If $S_{2}: l^{\prime} \times\left(l^{\prime}\right)^{N} \rightarrow l^{\prime}(X) \times\left(l^{\prime}\right)^{N}$ is the surjection which on $\left(l^{\prime}\right)^{N}$ is the identity and on $/$ is the quotient map onto $I^{\prime}(X)$, then $S=S_{1} S_{2}$ is a surjection of $(l)^{N}$ onto $E$ such that $\operatorname{ker} S=S_{2}^{-1}\left(I^{\prime}\right)$ is Banach. Now let $T:(I)^{N} \times\left(I^{N}\right)^{N} \rightarrow E$ be the surjection which is $S$ on the first copy of $(l)^{N}$ and is the zero map on the second copy. Then ker $T=\operatorname{ker} S \times(/)^{\mathrm{N}} \neq \operatorname{ker} S$.

Now we shall give some less direct consequences of Theorem 2.3 . 
Corollary 3.14. There are reflexive quojections $E_{1}, E_{2}, E_{3}$ having unconditional bases and containing subspaces $F_{1}, F_{2}, F_{3}$ respectively, such that:

(a) $F_{1}$, but not $E_{1} / F_{1}$, has an unconditional basis;

(b) $E_{2} / F_{2}$, but not $F_{2}$, has an unconditional basis:

(c) $F_{3}$ and $E_{3} / F_{3}$ have no tunconditional bases.

Proof. Follows from Corollaries 3.11, 3.12 and the result in [7]. Part (c) of the above corollary shows that the property of not having an unconditional basis is not a three-space property for the class of separable Fréchet spaces, thus providing the counterpart to Corollary 2.4 in [11].

At this point we camnot drop the word aunconditional" in the above statement, since twisted quojections may indeed have (conditional) bases, as shown in [13]. This, however, will be done in $\$ 4$.

Now, as in [1]], denote by SUM the class of countable direct sums of Banach spaces and call S-LB the class of strict (I.B)-spaces. Evidently, s-1.B $\backslash S U M$ is the class of twisted, strict (LB)-spaces (Cl. $[12, \S 1]$ ). Then, dualizing Corollaries 3.11 and 3.12 we obtain (for the amoreover parts use [6. Proposition 3.17).

Corollary 3.15. For $i=1,2,3$ there are reflexive $G_{i} \in S U M$ conlaining respertively suhspares $l_{i} \in \mathrm{s}-\mathrm{I}$. B such that $G_{i} / H_{i} \in \mathrm{s}-\mathrm{L}$. B and

(a) $H_{1} \in \mathrm{SUM}, G_{1} / H_{1} \notin \mathrm{SUM}$;

(b) $H_{2}\left(S \mathrm{SUM},\left(i_{2} / H_{2} \in \mathrm{SUM}\right.\right.$;

(c) $H_{3}, G_{3} / I_{3} \& S U \mathrm{M}$.

Moresuer, the analogte of corollary 3.14 holds.

Finally, it is perhaps superfluous to note that, by duality. (8), (9) and (10) yield corresponding exact sequences for reflexive spaces in s-1.B.

\section{APPLICATIONS TO NUCLEAR SPACES}

First of all we observe that, with reference to the construction of standard yuojections $\left.Q \mid\left(X_{n}\right),\left(Z_{n}\right),\left(s_{n}\right) ; L\right]$ outlined at the beginning of $\$ 2$, if the spaces 
$X_{n}, Z_{n}$ and $L$ are Fréchet spaces with continuous norms (each space having a fundamental system of norms fixed once and for all), then the same construction yields a Fréchet space without continuous norm which is twisted if and only if ker $s_{n}$ is not complement in $X_{n}$ for infinitely many $n$ (cfr. Lemma 2.1). The details of all this are left to the reader; but we point out that in the nuclear case the above construction was implicitly performed in $[17, \S 2]$. where it was explicitly carried out in the dual space. Here too we shall confine ourselves to the nuclear case and indicate how some of the results of $\$ \$ 2$ and 3 go over to this case. (Note that $\$ 1$ has no nuclear analogue, since there is no quotient-universal nuclear space).

The first result is the following analogue of Theorem 2.3.

Theorem 4.1. Let $\left(X_{n}\right),\left(Y_{n}\right)$ be two sequences of nuclear Fréchet spaces with continuous norms for which there exist surjections $r_{n}: X_{n} \rightarrow Y_{n}$. Further, let $\left(Z_{n}\right)$ be a sequence of nuclear Frecher spaces with continuous norms for which there are surjections $s_{n}: Z_{n} \rightarrow$ ker $r_{n}$. Then we have an exact sequence

$$
\begin{gathered}
0-Q\left[\left(Z_{n}\right),\left(\operatorname{ker} r_{n}\right),\left(s_{n}\right) ; L\right]-L\left(X_{n}\right) \times\left.\right|_{n} Z_{n} \rightarrow Q\left[\left(X_{n}\right),\right. \\
\left.\left(Y_{n}\right),\left(r_{n}\right) ; L\right] \rightarrow 0,
\end{gathered}
$$

from which the analogues of (9) and (10) may also be derived.

Proof. It suffices to note that the prools of Lemma 2.2 and Theorem 2.3 go over to this case.

Of course, Theorem 4.1 is not as far reaching as Theorem 2.3 so that. although Corollaries 3.1 and 3.3 have nuclear analogues, there is no hope of getting something like Corollaries 3.7 and 3.8 . However, we wish to draw attention to the following analogue of Corollaries 3.11 and 3.12 .

Let $\alpha=\left(\alpha_{n}\right)$ be an increasing sequence of positive real numbers such that

$$
\lim _{n} \frac{\log n}{\alpha_{n}}=0 \text { and } \sup _{n} \frac{\alpha_{n n^{2}}}{\alpha_{n}}<\infty
$$

and let $\Lambda_{1}=\Lambda_{1}(\alpha)$ be the associated nuclear power series space of finite type Then we have

Corollary 4.2. There is a surjection $\because \Lambda_{1} \rightarrow \Lambda_{1}$ such that the space $F=Q\left(\Lambda_{1}, \Lambda_{1}, r ; \Lambda_{1}\right)$ is rwisted. Moreover, we have the following thee eract sequences, nome of which splits: 


$$
\begin{aligned}
& 0 \rightarrow F \rightarrow \Lambda_{1}^{N} \rightarrow F \rightarrow 0, \\
& 0 \rightarrow \Lambda_{1} \rightarrow \Lambda_{1}^{N} \rightarrow F \rightarrow 0, \\
& 0 \rightarrow F \rightarrow \Lambda_{1}^{N} \rightarrow \Lambda_{1}^{N} \rightarrow 0 .
\end{aligned}
$$

Proof. Since $\Lambda_{1} \oplus \Lambda_{1} \simeq \Lambda_{1}$, by [18, Sat7. 5.4] there is an cxact sequence

$$
0 \rightarrow \Lambda_{1} \rightarrow \Lambda_{1} \stackrel{p}{\rightarrow} \Lambda_{1} \rightarrow 0
$$

with ker $r$ not complemented in $\Lambda_{1}$. Since ker $r \simeq \Lambda_{1}$, we have a surjection $s$ : $\Lambda_{1} \rightarrow \operatorname{ker} r$ with ker $s$ not complemented in $\Lambda_{1}$. Also, $\Lambda_{1}\left(\Lambda_{T}\right) \simeq \Lambda_{1} \otimes \Lambda_{1} \simeq \Lambda_{T}$ by $(12)_{2}$ and (13) follows from (11) by appropriately choosing everything. Similarly for (14) and (15). By [16, Theorem 2.3] none of the sequences splits.

Remark 4.3. We note the curious fact that all the steps in $Q\left(A_{1}, \Lambda_{1}, r\right.$; $\Lambda_{1}$ ) are isomorphic to $\Lambda_{1}$.

From Corollaty 4.2 (13) we immediately derive, via $[17, \$ 2]$ or $[6$, Proposition 4.1], the announced improvement of Corollary 3.14 (c).

Corollary 4.4. The property of not having a basis is not a three-space property for the class of separable (even nuclear) Fréchet spaces. The same is true also for the classes of separable (LB) - or (DF) - spaces.

Remark 4.5. The first assertion in the above corollary should be compared with the result in [14].

Finally, we observe that (14) also holds for nuclear power series spaces of infinite type. In lact, suppose thall $\alpha$ satisfies $(12)_{2}$ and sup $\log n / \alpha_{n}<\infty$ and put $\Lambda_{\infty}=\Lambda_{\infty}(\alpha)$. Then we have

Corollary 4,6. There is a muclear Fréchet space $Y$ (depending on $\alpha$ ) with a contimusus nom and surjection $\mathrm{r}: \Lambda_{\infty}-\gamma$ such that $Q\left(\Lambda_{\infty}, \gamma, r: \Lambda_{\infty}\right)$ is wisted and we have the follewing eract sequence (which does not split):

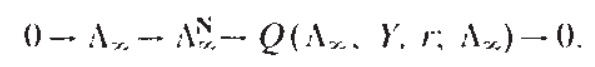


Proof. Let $\beta=\left(\beta_{n}\right)$ be an increasing sequence of positive real numbers satisfying (12), and $\lim \alpha_{n} / \beta_{n}=0$. By [21, Lemma 3.3] there is a subspace $\tilde{E}$ of $\Lambda_{\infty}$ and an exact sequence

$$
0 \rightarrow \Lambda_{\infty} \rightarrow \Lambda_{\infty} \stackrel{r}{\rightarrow} \Lambda_{1}(\beta) \oplus \tilde{E} \rightarrow 0
$$

Since $\Lambda_{1}(\beta)$ is not isomorphic to a complemented subspace of $\Lambda_{\infty}$, ker $r$ is not complemented in $\Lambda_{\infty}$ and the result follows by taking $Y=\Lambda_{1}(\beta) \oplus \tilde{E}$.

\section{References}

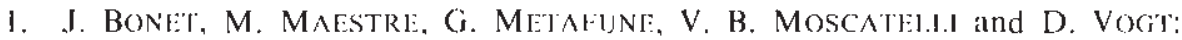
"Every guojection is the quotient of a countable product of Banach spaces", in Advances in the Theorl of Frechet Spaces (T. Terzioglu ed.). Kluwer Academic Publishers. 1989. 355-356.

2. J. BONEl and S. Dilkoti: "Fréchet spaces of Moscatelli typen, Re'v. Matem. Univ. Complutense Madrid 2 (suppl,) (1989), 77-92.

3. V. E. Cholonovskנ1: "On quasinormability of semimetrixable topological vector spacesm, Fumke. Anal. 7 (1976), 157-160.

4. M. DE WILDF: aSur le relivement des parties bornées d'un quotient d'espaces vectoriels topologiques". Bull. Soc. Rov. Sri. Liege 43 (1971). 299-301.

5. S. DIEROIf: and W. ROII.CKE: "On the three-space-problem for topological vector spaces", Collect Math. 32 (1981), 13-35.

6. K. FLorleT and V, B, MOSCATI:L,I; "On bases in strict inductive and projective limits of Iocally convex spaces". Pacific J. Math. 119 (1985), 103-113.

7. - : aUnconditional bases in Fréchet spaces", Arch. Math. 47 (1986), 129-130.

8. M. I. KADEC and A. PElcZYYNSK J: «Bases, lacunary sequences and complemented

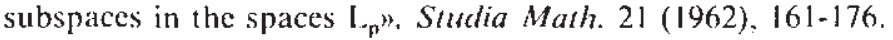

9. G. Köthl: “Topological Vector Spacess, I. Springer, Berlin, 1969.

10. J. LINDENSTRUSS and L. TZAFRIRI; "Classical Banach Spaces». [. If, Springer. Berlin, 1977, 1979

11. G. METAFuNe and V. B. Moscatel.1. «On the three-space property for locally convex spacesn. Colle't. Math. 37 (1986), 287-296.

12. -. : "Another construction of twisted spaces". Proc. Roy. Irish Acad. Sect. A $87(1987), 163-168$.

13. : "A twisted Frechet spatce with basism, Monatsh. Math. 105 (1988), 127-129.

14. : Nelclear Fréchet spaces with basis do not have the three-space property", Arch. Marh. 50 (1988), 369-370

15. : "Complemented subspices of sums and products of Banach spacess, Am. Mar. Pura Appl. 153 (1988). 175-190.

16. : "On lwisted Frechel and (1.B)-spaces". Proc. Amer. Math. Sere. 108 (1990), 145-150.

17. V. B. Mosicxtilit: aFrecthet spaces without continuous norms and without bascss. Bull. London Masth. Soss. 12 (1980). 6.3-66.

18. D. Vocial: "Line Charakterisierung der Eotenzreihenräume von endliches "lyp und ihre rolgerungen". Mamtseripta Math. 37 (1982), 269-301. 
19. - : "Some results on continuous linear maps between Fréchet spaces", in Funcional Analysis: Surveys and Recent Results /I/ (eds.: K. D. Bicrstedt and B. Fuchssteiner), North-Holland Math. Studies: 90, 1984, 349-381.

20. - : "On the functor's Ext" (E, F) for Frćchet spaces", Studia Math. 85 (1987), $163-197$.

21. D. VoGil and $M$. J. WAGNER: “Charakterisierung der Unterräume und Quotientenräume der nuklearen stabilen Potenzreihenräume von unendlichen Typ», Sructia Math. 70 (1981), 63-80.

Dipartinento di Matematica. Universita

C.P. 193. [-73100, Leece,

Recibido: 6 de julie de 1990

$$
\text { ltaty- }
$$

